\title{
Sasa-Satsuma equation: Soliton on a background and its limiting cases
}

\author{
U. Bandelow \\ Weierstrass Institute for Applied Analysis and Stochastics, Mohrenstraße 39, 10117 Berlin, Germany \\ N. Akhmediev \\ Optical Sciences Group, Research School of Physics and Engineering, Institute of Advanced Studies, The Australian National University, \\ Canberra ACT 0200, Australia
}

(Received 18 July 2012; published 27 August 2012)

\begin{abstract}
We present a multiparameter family of a soliton on a background solution to the Sasa-Satsuma equation. The solution is controlled by a set of several free parameters that control the background amplitude as well as the soliton itself. This family of solutions admits a few nontrivial limiting cases that are considered in detail. Among these special cases is the nonlinear Schrödinger equation limit and the limit of rogue wave solutions.
\end{abstract}

DOI: 10.1103/PhysRevE.86.026606

PACS number(s): 05.45.Yv, 42.65.Tg, 42.81.Dp

\section{INTRODUCTION}

Recent progress in the theory of integrable partial differential equations made a revolution in mathematics and expanded significantly the areas of physical application of these equations [1]. The Sasa-Satsuma equation [2] (SSE) is one of the integrable extensions of the nonlinear Schrödinger equation (NLSE). Although with fixed relation between higher order terms, it contains the most essential contributions often found in various physical applications: deep water waves $[3,4]$ and pulse propagation in optical fibers $[5,6]$. Namely, it contains the term with third order dispersion, the term with self-frequency shift, and the term describing self-steepening [7]. These are the most general terms that have to be taken into account when extending the applicability of the NLSE. According to the original work of Sasa and Satsuma [2] the equation can be written as

$$
i \psi_{\tau}+\frac{\psi_{x x}}{2}+|\psi|^{2} \psi+i \epsilon\left[\psi_{x x x}+3\left(|\psi|^{2}\right)_{x} \psi+6|\psi|^{2} \psi_{x}\right]=0 .
$$

Here, an arbitrary real parameter $\epsilon$ scales the integrable perturbations of the NLSE. When $\epsilon=0$, Eq. (1) reduces to the standard NLSE which has only the terms describing lowest order dispersion and self-phase modulation. This form of equation has been used in the series of works by Mihalache and co-workers [8-10]. There are a number of publications dealing with the solutions of the SSE [11-13]. Solutions with nonzero boundary conditions have been presented by Wright III [14] although the form of the SSE in his work and the technique used are different from the original version [2] and from the technique presented in Refs. [8-10].

In this paper, we are dealing with the solutions of Eq. (1) written in the form most common in applications. In particular, we present the soliton solution on a background which has been found to be important in the theory of rogue waves. Perhaps not the most general one but similar to the analogous solution of the NLSE [15,16], it contains important particular cases such as the breather solution periodic in the transverse direction, the zero velocity soliton solution, and the rogue wave solution.

One of the deficiencies of the previous works related to the SSE is the lack of descriptive illustrative material. Being mathematically highly nontrivial, derivations remain unused until we can see clearly the solutions in picture format. A selection of a few profiles chosen at fixed distances usually does not help when the soliton has complicated oscillatory behavior in propagation as in the SSE case. Modern style three-dimensional (3D) images help in appreciation of their complexity and in further use in applications. With this aim, we illustrate the general solution that we present as well as a few particular cases. The latter contains the rogue wave solution that is an important particular case by itself [17].

Solutions of Eq. (1) are nontrivial in many respects. First, there are several branches of solutions for the same set of parameters. We do not try to consider all of them in one work. A better answer is to concentrate the attention on just one branch and see the variety of possibilities within it. Another difficulty of operating with solutions of each branch is considering limiting cases. They are not simple either. Each particular case requires considering the limits carefully. Even the limit of zero background which seems to be simple in the NLSE case is quite involved when we are dealing with the solutions of the SSE.

One of the constraints used in Ref. [14] to obtain the solutions is fixed $\epsilon$. This is a significant restriction especially when we are interested in physical extensions of solutions of the NLSE. In most of the problems of practical interest the last three terms are usually small in comparison with the basic NLSE terms. Thus, it is important to keep the parameter $\epsilon$ arbitrary and, in particular, consider the limit of small values of $\epsilon$. Ignoring this possibility, we may end up with singularities [14] and have the impression that the SSE solutions cannot be considered as an extension of those for the NLSE.

In order to extend the solutions with fixed $\epsilon$ to include arbitrary $\epsilon$, we recall that there is a scaling transformation of the NLSE [18]. Namely, if we have a particular solution of the NLSE $\psi(x, t)$, a more general solution $\tilde{\psi}(x, t)$ with a free parameter $\mu$ can be obtained via the transformation:

$$
\tilde{\psi}(x, \tau ; \mu)=\mu \psi\left(\mu x, \mu^{2} \tau\right) .
$$

Applying the same transformation to Eq. (1) shows that it transforms the Sasa-Satsuma equation into

$$
\begin{aligned}
& i \tilde{\psi}_{\tau}+\frac{1}{2} \tilde{\psi}_{x x}+|\tilde{\psi}|^{2} \tilde{\psi}+i \epsilon \mu\left(\tilde{\psi}_{x x x}\right. \\
& \left.\quad+3\left(|\tilde{\psi}|^{2}\right)_{x} \tilde{\psi}+6\left(|\tilde{\psi}|^{2}\right) \tilde{\psi}_{x}\right)=0 .
\end{aligned}
$$


We notice that choosing $\mu=1 / \epsilon$ eliminates $\epsilon$ from the equation. This is an important observation as with the help of the transformation we can use the solutions $\psi_{d}(x, \tau)$ developed for a fixed value of $\epsilon=1$, and keep the parameter $\epsilon$ arbitrary by setting

$$
\psi(x, \tau)=\frac{1}{\epsilon} \psi_{d}\left(\frac{x}{\epsilon}, \frac{\tau}{\epsilon^{2}}\right) .
$$

In this way, the parameter $\epsilon$ can be incorporated into any solution that has been obtained originally for $\epsilon$ fixed to a certain value. We note that Eq. (4) indeed makes an impression that the solution is singular when $\epsilon \rightarrow 0$. However, this problem can be fixed with a suitable choice of other free parameters of the solution. Let us illustrate this point using a simple example.

As we are interested in solutions on a background, we start with the plane-wave solutions of the Sasa-Satsuma equation in the form

$$
\psi_{0}(x, \tau)=\frac{c}{2 \epsilon} \exp \left[-\frac{i}{2 \epsilon}\left(k x-\frac{\omega}{4 \epsilon} \tau\right)\right],
$$

where the amplitude $c$, the wave number $k$, and the frequency $\omega$ are related through

$$
\omega=6 c^{2} k+2 c^{2}-k^{3}-k^{2} .
$$

We can notice that in here, the coefficient $\epsilon$ is already included into the solution. It again looks singular at the limit $\epsilon \rightarrow 0$. However, the correct choice of parameters $c \sim \epsilon, \omega \sim \epsilon^{2}$, and $k \sim \epsilon$ eliminates this singularity and in the $\epsilon \rightarrow 0$ limit, we obtain the plane wave of the NLSE with finite amplitude.

Below, we present soliton solutions of the SSE on a background. This is a multiparameter solution with variable background, arbitrary velocity for arbitrary real $\epsilon$. As a result, there are several limiting cases that can be calculated using the general expression. Usually, solitons on a background are pulsating formations for the NLSE. This happens due to the nonlinear interference between the soliton and the background that have different propagation constants [19]. The same can be said about the solitons on a background for the SSE. They are oscillating along the direction of propagation. This particular oscillation disappears in the limit of zero background. This special limit contains soliton solutions that have been obtained earlier [2]. We provide this correspondence explicitly.

\section{SOLITON ON A BACKGROUND}

The technique we use is similar to the one employed in Ref. [14]. We omit this cumbersome part and just present the solution that can be checked using any modern software with symbolic computation facilities. We stress that this is just one branch of solutions. However, it contains most important particular cases. The one-soliton solution on a background $\psi(x, \tau)$ of Eq. (1) is given by

$$
\psi(x, \tau)=\psi_{0}(x, \tau)\left[1+\frac{i\left(\zeta-\zeta^{*}\right) G(x, \tau)}{c|\zeta|^{2} f_{3}(x, \tau)}\right]
$$

with

$$
\begin{aligned}
G(x, \tau)= & {\left[\zeta^{*} f_{1}(x, \tau)+\zeta f_{2}^{*}(x, \tau)\right] } \\
& \times\left[\zeta\left|f_{1}(x, \tau)\right|^{2}+\zeta^{*}\left|f_{2}(x, \tau)\right|^{2}\right] \\
& +\frac{1}{2}\left(\zeta+\zeta^{*}\right)\left[\zeta f_{1}(x, \tau)+\zeta^{*} f_{2}^{*}(x, \tau)\right],
\end{aligned}
$$

where

$$
\begin{gathered}
f_{1}(x, \tau)=\frac{r_{11}+r_{12} \Gamma \exp (i M x+i N \tau)}{1+\Gamma \exp (i M x+i N \tau)} \\
f_{2}(x, \tau)=\frac{r_{21}+r_{22} \Gamma \exp (i M x+i N \tau)}{1+\Gamma \exp (i M x+i N \tau)} \\
f_{3}(x, \tau)=\left(1+\left|f_{1}\right|^{2}+\left|f_{2}\right|^{2}\right)^{2}+\frac{\left(\zeta-\zeta^{*}\right)^{2}}{4|\zeta|^{2}}\left|1+2 f_{1} f_{2}\right|^{2} \\
M=m_{2}-m_{1}
\end{gathered}
$$

and

$$
r_{n j}=\frac{-i 3 c}{3 m_{j}+(-1)^{n} K-\zeta},
$$

where indices $n, j=1,2$ and $K=1+3 k$. Complex conjugation is denoted by the star $z^{*}$ throughout this paper. The parameter $\zeta \in \mathbb{C}$ here is the complex eigenvalue of the spectral problem while $\Gamma$ is another arbitrary complex number which is related to ordinary translations $x_{0}$ and $\tau_{0}$ of the solution along the $x$ and $\tau$ axes. This can be easily seen if we write $\Gamma$ in the form $\Gamma=\exp \left(-i M x_{0}-i N \tau_{0}\right)$.

The spatial eigenvalues $m_{j}$ in Eqs. (8) and (9) are two of the solutions of the third order polynomial

$$
\begin{aligned}
& m^{3}-m\left[2 c^{2}+\frac{\zeta^{2}}{3}+\left(\frac{K}{3}\right)^{2}\right] \\
& +\frac{2}{3} \zeta\left[c^{2}-\left(\frac{K}{3}\right)^{2}\right]+\frac{2 \zeta^{3}}{27}=0
\end{aligned}
$$

such that each $m_{j}$ depends on the free parameters, i.e., $m_{j}=$ $m_{j}(\zeta, c, k)$. In this work, we operate with one of the Riemann surfaces of the solution and choose the pair of $m_{j}$ that have similar form:

$$
\begin{aligned}
& m_{1}=\frac{i\left[\sqrt[3]{3}(-\sqrt{3}+i) u_{1}+(\sqrt{3}+i) u_{9}^{2}\right]}{63^{2 / 3} u_{9}}, \\
& m_{2}=\frac{i\left[\sqrt[3]{3}(\sqrt{3}+i) u_{1}+(-\sqrt{3}+i) u_{9}^{2}\right]}{63^{2 / 3} u_{9}},
\end{aligned}
$$

where

$$
\begin{aligned}
& u_{1}=18 c^{2}+3 \zeta^{2}+K^{2}, \quad u_{2}=9 c^{2}+\zeta^{2}-K^{2}, \\
& u_{5}=18 c^{2}+\frac{1}{3}\left(4-K^{2}\right), \quad u_{6}=36 c^{2}-K^{2}+3, \\
& u_{7}=18\left(1-9 c^{2}\right) \zeta^{3}+2 \zeta u_{6}\left(18 c^{2}+K^{2}\right)-54 \zeta^{5}, \\
& u_{9}=\sqrt[3]{\sqrt{3} \sqrt{27 \zeta^{2} u_{2}^{2}-u_{1}^{3}}-9 \zeta u_{2},} \\
& u_{4}=27 \zeta^{4}-27 \zeta^{2} u_{5}+u_{6}^{2}, \quad u_{0}=u_{6}-6 \zeta^{2} .
\end{aligned}
$$

Using these expressions, we can specify the spatial frequency $M=m_{2}-m_{1}$ :

$$
M=-\frac{i\left(u_{9}^{2}-\sqrt[3]{3} u_{1}\right)}{6 \sqrt[6]{3} u_{9} \epsilon},
$$

and the temporal frequency $N$ :

$$
N=\frac{3\left(3 \zeta u_{7}-u_{0} u_{4}\right) M}{216 \epsilon^{2}\left(u_{0}-9 \zeta m_{2}\right)\left(u_{0}-9 \zeta m_{1}\right)} .
$$

The solution (7) $\psi(x, \tau ; c, k, \zeta ; \Gamma, \epsilon)$ is thereby completely determined by the parameters of the plane wave $\psi_{0}(5)$ with $c$, $\omega$, and $k$, restricted by Eq. (6), and $\zeta \in \mathbb{C}$. 


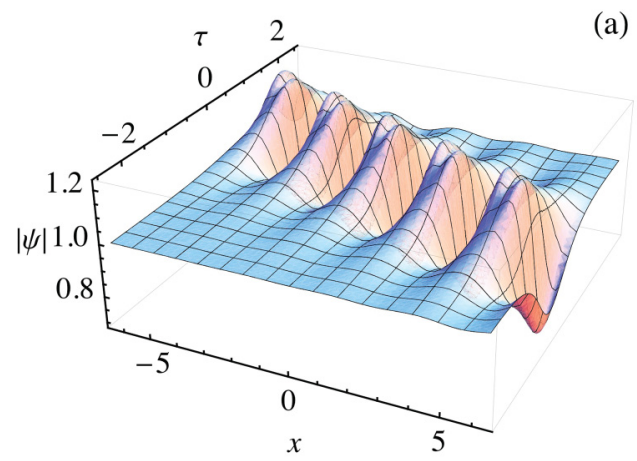

(a)

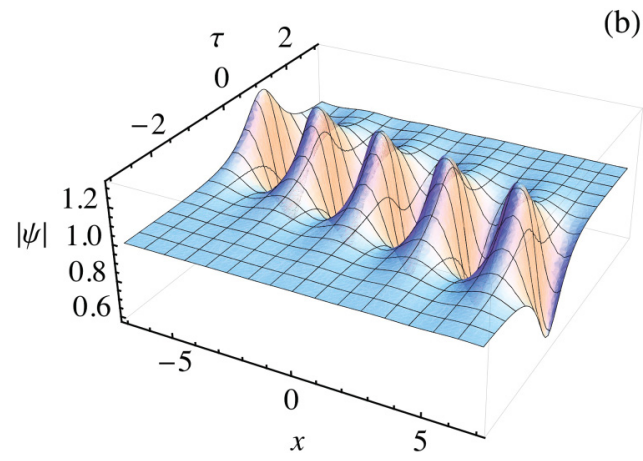

FIG. 1. (Color online) Soliton solution on a background defined by Eq. (7). Parameters are $\epsilon=1 / 2, \zeta=1.5+i 0.5, c=1, \Gamma=1$. (a) $k=0.1(K=1.3)$, resulting in $M=-2.2334-i 0.325551$ and $2 N=1.99665-i 3.88523$; (b) $k=0.6(K=2.8)$ resulting in $M=$ $-2.024-i 0.231356$ and $2 N=-0.6966-i 4.31113$.

An interesting observation is that the solution (7) is periodic along each of the parallel set of lines,

$$
\operatorname{Im}[M x+N \tau+\text { const }]=0
$$

i.e., along the direction of propagation, where the imaginary part, denoted by "Im[z]," of the above expression vanishes. This follows from the form of exponential functions in Eqs. (8) and (9). The phase and location of the periodic function is given by $\Gamma$. Two typical examples of the solution are shown in Figs. 1(a) and 1(b). Here, the background plane wave is controlled by the amplitude $c /(2 \epsilon)$, and the direction of propagation (phase velocity) $4 \epsilon k / \omega$. The solitonic part of the solution is controlled by the complex eigenvalue $\zeta$. In particular, it defines the amplitude, velocity, and periodicity of the soliton. Each peak within the soliton can have double or single maximum depending on the combination of all parameters. In the particular case shown in Fig. 1(a) each peak has a double maximum while in Fig. 1(b) each peak has a single maximum. Qualitatively, this solution is similar to the NLSE solution presented in Fig. 7 of Ref. [16].

\section{SPATIALLY PERIODIC BREATHER ON A BACKGROUND}

For solutions which are periodic along the $x$ axis the period is defined by $M$ which has to be real. The condition of zero imaginary part of $M$ leads to the eigenvalues of inverse scattering $\zeta$ being given by

$$
\zeta= \pm \sqrt{\alpha \pm \beta}
$$

with

$$
\begin{aligned}
& \alpha=\frac{c^{4}+10 c^{2}\left[\left(\frac{K}{3}\right)^{2}-\frac{3 M^{2}}{10}\right]+2 Q\left[M^{2}+\left(\frac{K}{3}\right)^{2}\right]}{2 Q}, \\
& \beta=\frac{2 w}{3 Q}\left[\frac{1}{4}\left(c^{2}+M^{2}\right)-\left(\frac{K}{3}\right)^{2}\right],
\end{aligned}
$$

where $w=\sqrt{9 c^{4}+K^{2}\left(M^{2}-4 c^{2}\right)}$ and $Q=\frac{M^{2}}{4}-\left(\frac{K}{3}\right)^{2}$. Any choice of signs in Eq. (17) provides us with a valid complex eigenvalue. In all four cases $\zeta$ is a function of $c, k$, and $M$. When we choose both signs in Eq. (17) to be positive, we obtain, explicitly,

$$
\begin{aligned}
\zeta= & \frac{1}{3} \sqrt{\frac{1}{4 K^{2}-9 M^{2}}} \times\left[-162 c^{4}-18 c^{2}\left[3\left(w-9 M^{2}\right)+10 K^{2}\right]\right. \\
& \left.+\left(4 K^{2}-9 M^{2}\right)\left(6 w+9 M^{2}+K^{2}\right)\right]^{1 / 2} .
\end{aligned}
$$

The breather solution in this case is periodic in $x$ and has a single growth-decay cycle along the $\tau$ axis. It starts with modulation instability, grows to maximum amplitude, and decays the same way as it grew. This solution is similar to the Akhmediev breather solution of the NLSE [20-22]. However, there are more parameters to play with in the SSE case.

Two examples of the breather profiles are shown in Fig. 2. For the chosen set of parameters $c=1$ and $\epsilon=1 / 2$ the

(a)

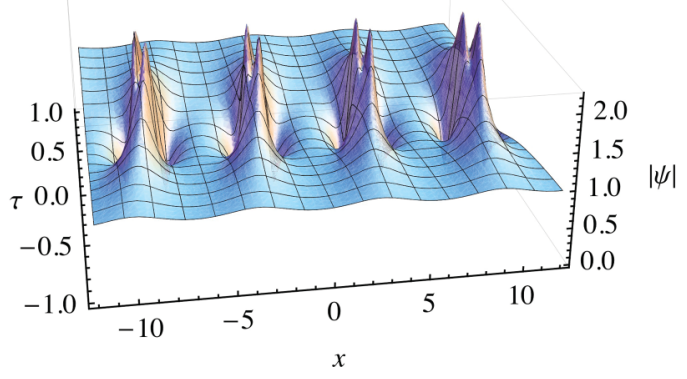

(b)

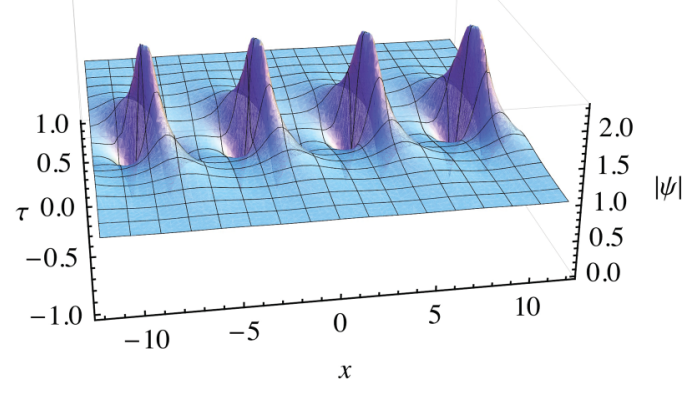

FIG. 2. (Color online) Breather solution of SSE defined by Eq. (7) becomes spatially periodic for purely real $M$. Parameters used in this case are $\epsilon=1 / 2, c=1, M=1$. In (a) $k=1(K=4)$, resulting in $\zeta=0.957906+i 1.81754$ and $2 N=3-i 6.245$. In (b), $k=1.9$ $(K=6.7)$ resulting in $\zeta=2.00943+i 1.76148$ and $2 N=-6.63-$ $i 11.2103$. In each case, $\Gamma=+1$. 
background amplitude is 1 . The parameter $M$ defines the period of the structure. For $k=1$, like in Fig. 2(a), each maximum of the breather profile has a double peak structure while for $k=1.9$, like in Fig. 2(b), the two peaks join into a single one.

\section{TEMPORAL PERIODIC SOLITONS ON A BACKGROUND}

The temporal frequency $N$ contains the real part (frequency $\lambda)$ and an exponential decay coefficient $\nu$,

$$
N=\lambda+i \nu
$$

To obtain purely periodic solutions along the $\tau$ axis, $N$ must be purely real, i.e., $v=0$. Parameters for this condition to be fulfilled can be found numerically. An example of such a solution is shown in Fig. 3. The parameter $\zeta$ for this example calculated numerically along with other parameters is given in the caption to Fig. 3. In this example, the solution has a double peak structure. This can be seen from the fact that the adjacent maxima in the figure are slightly shifted relative to the center line of the soliton. Choosing a different set of parameters, we can obtain a solution with a single peak structure. This solution is analogous to the Kuznetsov-Ma soliton of the NLSE [23].

\section{ROGUE WAVE SOLUTION}

If we take the long period limit of the solution shown in Fig. 2 and use a specific value of $\Gamma=-1$ required to keep the

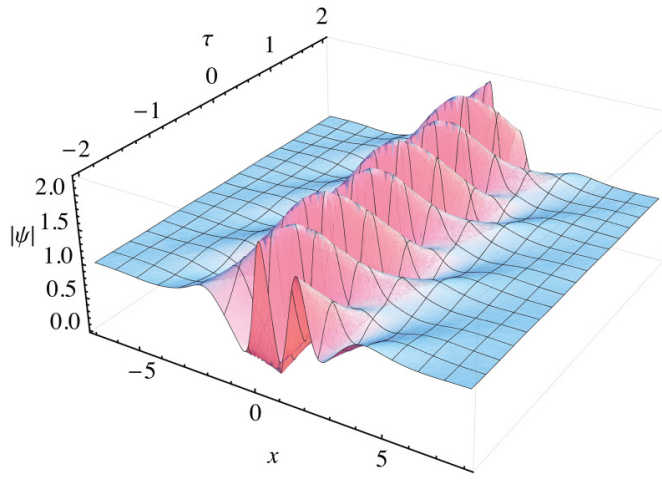

FIG. 3. (Color online) Soliton solution on a background for the case of purely real $N$. The profile is given by Eq. (7) with the following parameters: $\epsilon=1 / 2, k=0.1(K=1.3), c=1$, resulting in $\zeta=0.5+i 1.672, M=-1.38258-i 0.806851, \lambda=19.48924$, and $\Gamma=1$.

central maximum close to the origin, we are left with just one infinite period. This way we obtain the solution describing the rogue wave of the SSE (1):

$\psi(x, \tau)=\frac{c}{2 \epsilon}\left(1-\frac{\zeta-\zeta^{*}}{c} G\right) \exp \left[-i\left(\frac{k}{2 \epsilon} x-\frac{\omega}{8 \epsilon^{2}} \tau\right)\right]$,

with $\omega$ given by Eq. (6) and $G$ given by

$$
G=\frac{|u|^{2} \operatorname{Re}[\zeta]\left(\zeta u^{*} g+\zeta^{*} u h^{*}\right)+\left(\zeta|g|^{2}+\zeta^{*}|h|^{2}\right)\left(\zeta^{*} u^{*} g+\zeta u h^{*}\right)}{|\zeta|^{2}\left(|u|^{2}+|g|^{2}+|h|^{2}\right)^{2}-\left|u^{2}+2 h g\right|^{2} \operatorname{Im}[\zeta]^{2}}
$$

where

$$
\begin{aligned}
\zeta & = \pm \frac{i \sqrt{9 c^{2}\left(9 c^{2}+10 K^{2}\right)+3 c\left(9 c^{2}-4 K^{2}\right)^{3 / 2}-2 K^{4}}}{3 \sqrt{2} K}, \\
u & =(\chi \tau+2 \epsilon x), \quad h=3 c\left(\frac{u}{M_{1}}+i \frac{12 \epsilon^{2}}{M_{1}^{2}}\right), \\
g & =3 c\left(\frac{u}{M_{2}}-i \frac{12 \epsilon^{2}}{M_{2}^{2}}\right), \quad M_{1}=K+d-\zeta, \\
M_{2} & =K-d+\zeta, \quad d=\left(\frac{b}{2}+\frac{2 u_{1}}{3 b}\right), \\
b & =(-1+i \sqrt{3})\left[-\zeta u_{2}\right]^{1 / 3}, \quad \\
\chi & =\frac{9\left(a-6 c^{2}\right) \zeta^{4}+3 a\left(a-1-18 c^{2}\right) \zeta^{2}+a^{3}}{6\left(2 \zeta^{2}+d \zeta+a\right)^{2}}, \quad a=-\frac{u_{6}}{3} .
\end{aligned}
$$

The solution depends on variables $x$ and $\tau$, as well as on three real parameters $\epsilon, K=1+3 k$, and $c$.

Comparison of this rogue wave solution to the one of the NLSE [24] or Hirota [25] equations shows that the SSE rogue wave has a significantly more complicated structure. In particular, it involves polynomials of fourth order rather than second order as in the two previous cases. This can be seen from the structure of the expression (21) with the nominator and denominator being of fourth power of $u$.

Rogue waves do exist provided that $9 c^{2}-4 K^{2}<0$. This follows from the requirement for the eigenvalue $\zeta$ of the spectral problem to have a nonzero real part. This happens when $|1+3 k|>3 c / 2$. Explicitly, the condition is either

$$
k>\frac{c}{2}-\frac{1}{3} \quad \text { or } \quad k<-\frac{c}{2}-\frac{1}{3} .
$$

Thus, the wave number $k$ can be zero only when $c<2 / 3$. Otherwise, the plane wave propagation direction has to be skewed for the rogue wave to exist.

The solution (20) is illustrated in Fig. 4 for the values of parameters $c=1, \epsilon=0.5$, and $k=0.8$. It exhibits a double peak and has a maximum amplitude of around 2.5. The background amplitude is $c /(2 \epsilon)$, which is equal to 1 here. For $c=1$ and any $\epsilon$, the wave number $k$ has to be larger than $1 / 6$. Thus, the plane wave propagates at an angle to the $\tau$ axis. The solution itself is also tilted. The solution keeps a double peak structure at all values of $k$ in the interval $1 / 6<k \lesssim 2$. However, at larger values of $k$ the two maxima merge and the solution has a single peak. An example is shown in Fig. 5 where $k=2$. 


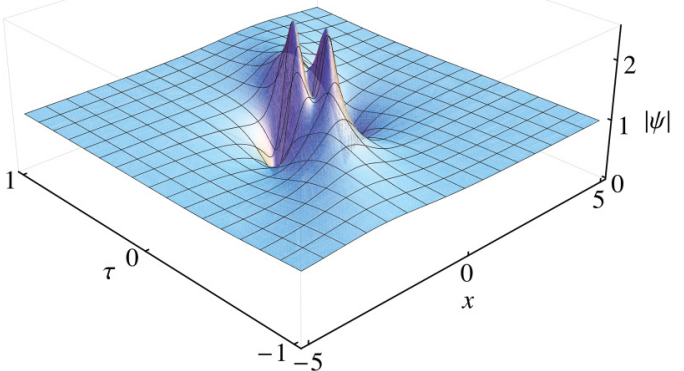

FIG. 4. (Color online) Rogue wave of the Sasa-Satsuma equation when $c=1, \epsilon=0.5$, and plane wave number $k=0.8$.

Despite seemingly singular structure of the solution with $\epsilon$ being in the denominator, the amplitude of the background for the rogue wave is finite and equal to $\psi_{0}=c /(2 \epsilon)$ just as in Eq. (5). We can keep it to be constant having the ratio $c / \epsilon$ to be a constant. When taking the limit $\epsilon \rightarrow 0$, we should simultaneously take the limit of $c \rightarrow 0$. Then $k$ and $\omega$ should also be considered in the same zero limit. This should be done carefully, to keep $k$ in the limits (22). As a result, when reducing the parameter $\epsilon$ to zero, and having the plane wave number $k=0$, the limit is the Peregrine solution of the NLSE [17].

\section{ZERO BACKGROUND LIMIT}

The background of solution (7) $c /(2 \epsilon)$ is controlled by the parameter $c$. The limit with zero background is obtained when $c \rightarrow 0$. The limit is far from being trivial. The difficulty is in finding the limits of the $m_{j}$ values that enter, among others, the $r_{n j}$ coefficients. These can be calculated using a series expansion of $m_{j}$ at small $c$ :

$$
r_{n j}=\frac{-i c}{m_{j}^{(0)}+(-1)^{n} \frac{K}{3}-\frac{\zeta}{3}+m_{j}^{(2)} c^{2}},
$$

where $m_{j}^{(0)}=\lim _{c \rightarrow 0} m_{j}$ and $m_{j}^{(2)}=\left.\frac{\partial^{2} m_{j}}{\partial c^{2}}\right|_{c=0}$.

In the limit $c \rightarrow 0$, the $m_{j}$ coefficients become

$$
\begin{aligned}
& m_{1}^{(0)}=-\frac{(\sqrt{3}+i) R^{+}+(\sqrt{3}-i)(\sigma)^{2 / 3} \sigma_{2}^{2} R}{6 \sqrt{3} \sqrt[3]{\sigma} \sigma_{2}}, \\
& m_{2}^{(0)}=i \frac{\left[R^{+}-(\sigma)^{2 / 3} \sigma_{2}^{2} R\right]}{3 \sqrt{3} \sqrt[3]{\sigma} \sigma_{2}},
\end{aligned}
$$

where

$$
\begin{gathered}
R=(K-i \sqrt{3} \zeta \sigma), \quad R^{+}=(K+i \sqrt{3} \zeta \sigma), \\
\sigma=\operatorname{sgn}\left[\operatorname{Im}\left(\zeta^{2} K\right)\right], \\
\sigma_{2}=\left\{\begin{array}{ccc}
e^{2 i \pi / 3} & \Leftrightarrow & -\frac{\pi}{3}>\operatorname{Arg}\left(w_{3 r}\right)>-\pi, \\
e^{-2 i \pi / 3} & \Leftrightarrow & \pi>\operatorname{Arg}\left(w_{3 r}\right)>\frac{\pi}{3}, \\
1 & \text { else }
\end{array}\right. \\
w_{3 r}=\frac{(\sqrt{3}+i)}{2} \sqrt[6]{3} \sqrt[3]{\sigma} R .
\end{gathered}
$$

Hence, various branches given above should be considered, which provide different limits for the $r_{n j}$ coefficients. Here, we

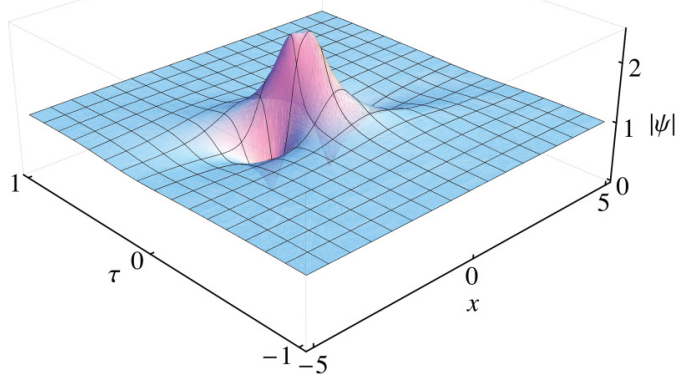

FIG. 5. (Color online) Rogue wave of the Sasa-Satsuma equation when $c=1, \epsilon=0.5$, and plane wave number $k=2$.

restrict ourselves in one of these branches given by $\sigma=+1$ and $\sigma_{2}=+1$. This requires the following two conditions:

(1) $\operatorname{sgn}\left[\operatorname{Im}\left(\zeta^{2} K\right)\right]=1$,

(2) $-\pi / 3<\arg \left[\sqrt[6]{-3} K-(-3)^{2 / 3} \zeta\right]<\pi / 3$.

In this case, the second order coefficients $m_{1}^{(2)}, m_{2}^{(2)}$ in the expression (23) are

$$
\begin{aligned}
& m_{1}^{(2)}=-\frac{3}{K-3 \zeta}, \\
& m_{2}^{(2)}=\frac{18 \zeta}{K^{2}-9 \zeta^{2}} .
\end{aligned}
$$

The spatial frequency $M^{(0)}=m_{2}^{(0)}-m_{1}^{(0)}$ then becomes

$$
M^{(0)}=\frac{K}{3}-\zeta .
$$

The corresponding temporal frequency $N^{(0)}=N_{2}^{(0)}-N_{1}^{(0)}$ is

$$
N^{(0)}=\frac{K}{6}\left(\frac{K^{2}}{9}-\frac{1}{3}\right)-\frac{\zeta}{2}\left(\zeta^{2}-\frac{1}{3}\right) .
$$

For brevity, we denote the exponential function appearing in Eq. (7) as $A$ :

$$
A=i(M x+N \tau)=\frac{i}{2 \epsilon}\left(M^{(0)} x+\frac{N^{(0)}}{\epsilon} \tau\right) .
$$

Along the branch considered here this function takes the form

$$
\begin{aligned}
A= & \frac{x}{2 \epsilon}\left[\eta+i\left(\frac{K}{3}-\xi\right)\right]-\frac{\tau}{8 \epsilon^{2}}\left[\eta\left(\eta^{2}-3 \xi^{2}+\frac{1}{3}\right)\right. \\
& \left.-i \xi\left(3 \eta^{2}-\xi^{2}+\frac{1}{3}\right)-i \frac{K}{3}\left(\frac{K^{2}}{9}-\frac{1}{3}\right)\right]
\end{aligned}
$$

with the real and the imaginary parts of $A$ given explicitly by setting $\zeta=\xi+i \eta$. The leading contributions at small $c$ are

$$
\begin{gathered}
r_{11}=\frac{3 i c}{2 K}, \quad r_{12}=\frac{3 i c}{3 \zeta+K}, \\
r_{21}=\frac{i(K-3 \zeta)}{3 c}, \quad r_{22}=\frac{3 i c}{3 \zeta-K} .
\end{gathered}
$$

Motivated by these expressions we introduce the following notations:

$$
Y=(3 \zeta-K) / 3, \quad W=(3 \zeta+K) / 3, \quad S=W Y,
$$

which will be used in formulas below. 
Within the approximations considered above, the form of the solution can be written explicitly in terms of $c$ :

$$
\begin{aligned}
\psi(x, \tau)= & \frac{1}{2 \epsilon} \exp \left[-\frac{i}{2 \epsilon}\left(k x-\frac{\omega}{4 \epsilon} \tau\right)\right] \\
& \times\left[c+\frac{i\left(\zeta-\zeta^{*}\right)}{|\zeta|^{2}} \frac{G(x, \tau)}{f_{3}(x, \tau)}\right],
\end{aligned}
$$

where in the lowest order of $c$

$$
\frac{G(x, \tau)}{f_{3}(x, \tau)}=-2 i c|\zeta|^{2} K W^{*} Y \frac{G_{0}}{f_{30}+f_{32} c^{2}}
$$

with

$$
\begin{aligned}
& G_{0}=-8|\zeta|^{2}|S|^{2}|Y|^{2} K^{3} W\left(Y^{*}\right)^{2}\left(e^{A} \Gamma+1\right) \\
& f_{30}=16 K^{4}|\zeta|^{2}|Y|^{4}|S|^{4} \\
& f_{32}=32 K^{4}|\zeta|^{2}|S|^{4}\left(\left|Y\left(e^{A} \Gamma+1\right)\right|^{2}-2 \operatorname{Re}\left[\left(Y^{*}\right)^{2} \Gamma e^{A}\right]\right)
\end{aligned}
$$

and where "Re$[z]$ " means the real part of $z$. The solution then can be written in a simpler form:

$$
\begin{aligned}
& \psi(x, \tau) \\
& =\frac{1}{2 \epsilon} \exp \left[-i\left(\frac{k}{2 \epsilon} x-\frac{\omega}{8 \epsilon^{2}} \tau\right)\right] \\
& \quad \times\left[c-\frac{\left(\zeta-\zeta^{*}\right) c Y^{*}|Y|^{2}\left(e^{A} \Gamma+1\right)}{|Y|^{4}+2 c^{2}\left\{\left|Y\left(e^{A} \Gamma+1\right)\right|^{2}-2 \operatorname{Re}\left[e^{A} \Gamma\left(Y^{*}\right)^{2}\right]\right\}}\right] .
\end{aligned}
$$

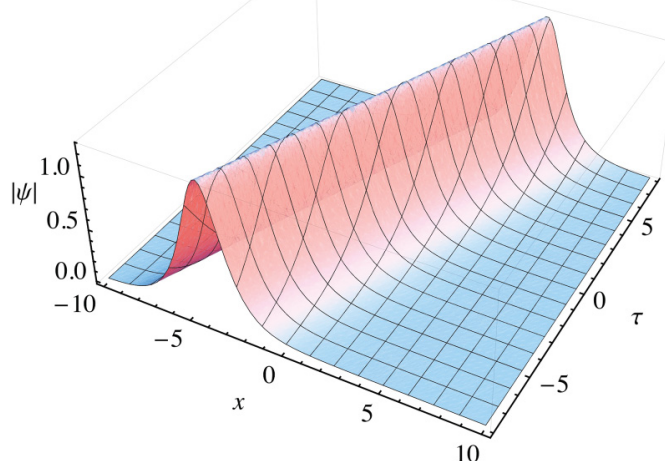

FIG. 6. (Color online) Amplitude profile of the solution (34) in the $(x, \tau)$ plane. Parameters chosen for this illustration are $c=10^{-3}, K=$ $1.3(k=0.1), \zeta=1 / 3+i(\xi=1 / 3, \eta=1), \epsilon=0.5$, and $\Gamma=1$.

The solution (34) is a fundamental sech-shaped soliton. It is illustrated in Fig. 6. When decreasing $c$, the shape and the height of the soliton does not change. However, it moves in the $(x, \tau)$ plane. This can be seen if we replace $c$ by $c=e^{-\rho}$ and notice that the variable $\rho$ adds up to the real part of the exponent $A$, causing the shift along the $x$ and $\tau$ variables.

In the next order of $c$ we include the term $\sim G_{2} c^{2}$ in the nominator and the term $\sim f_{34} c^{4}$ in the denominator:

$$
\frac{G(x, \tau)}{f_{3}(x, \tau)}=-2 i c|\zeta|^{2} K W^{*} Y \frac{G_{0}+G_{2} c^{2}}{f_{30}+f_{32} c^{2}+f_{34} c^{4}},
$$

where

$$
\begin{aligned}
G_{2}= & 4 K^{2}\left(\zeta^{*} Y^{*}\right)^{2}|W|^{2} \times\left\{\zeta \exp \left(A^{*}\right) \Gamma^{*}\left(4 K W Y^{2}-3|W|^{2}|Y|^{2}\right)-2 e^{A} \Gamma K\left[|Y|^{2}\left(2|\zeta|^{2}+\zeta W\right)-\zeta W\left(Y^{*}\right)^{2}\right]\right. \\
& -W|Y|^{2}\left(3 Y \zeta^{*}+2 K \operatorname{Re}[\zeta]\right)+4 \zeta W K e^{A} \Gamma \operatorname{Re}\left[e^{A} \Gamma\left(Y^{*}\right)^{2}\right]-2|Y|^{2} e^{A} \Gamma K\left\{\zeta \exp ^{\left(A^{*}\right)} \Gamma^{*}\left(2 \zeta^{*}+W\right)+\operatorname{Re}\left[e^{A} \Gamma \zeta W\right]\right\} \\
& \left.-2 \Gamma K W e^{2 \operatorname{Re}[A]+A} \operatorname{Re}[\zeta]|\Gamma|^{2}|Y|^{2}\right\}
\end{aligned}
$$

and

$$
\begin{aligned}
f_{34}= & 4 K^{2}|W|^{2} \times\left\{2|\zeta|^{2}\left(2 K^{2}+9 \operatorname{Re}\left[Y^{2}\right]\right)|W|^{2}|Y|^{4}+K\left|4 W^{2} Y\right|^{2}\left(\operatorname{Re}\left[e^{A} \Gamma \zeta^{*}\left(\zeta K-3 \operatorname{Im}[\zeta]^{2}\right)\right]+\frac{3}{2} \zeta^{2} \operatorname{Re}\left[e^{A} \Gamma W^{*}\right]\right)\right. \\
& +|4 K W Y|^{2} \operatorname{Re}\left[e^{A} \Gamma Y W^{*}\left(\operatorname{Im}[\zeta]^{2}-|\zeta|^{2} W^{*}\right)\right]+8 K^{2} e^{2 \operatorname{Re}[A]}|\Gamma|^{2}|Y|^{2}\left(|Y|^{2} \operatorname{Re}\left[\zeta^{2}|Y|^{2}-4 \operatorname{Im}[\zeta]^{2} Y W^{*}\right]\right. \\
& \left.+2|W|^{2}\left\{|Y|^{2}\left(\operatorname{Re}[\zeta]^{2}+|\zeta|^{2}\right)-\operatorname{Re}\left[Y^{2}\right]|\zeta|^{2}\right\}\right)-24 K|W|^{2} \operatorname{Re}\left[e^{2 A} \Gamma^{2} \zeta^{*} \operatorname{Im}[\zeta]^{2} W^{2}\left(Y^{*}\right)^{2}\right]-16 K^{2} e^{2 \operatorname{Re}[A]}|\Gamma|^{2}|Y|^{2} \\
& \left.\times\left(\operatorname{Im}[\zeta]^{2}|Y|^{2} \operatorname{Re}\left[e^{A} \Gamma W Y^{*}\right]+|W|^{2}\left\{|\zeta|^{2} \operatorname{Re}\left[e^{A} \Gamma\left(Y^{*}\right)^{2}\right]-\operatorname{Re}[\zeta]^{2} \operatorname{Re}\left[e^{A} \Gamma\right]|Y|^{2}\right\}\right)+4 K^{2} e^{4 \operatorname{Re}[A]} \operatorname{Re}[\zeta]^{2}|\Gamma|^{4}|W|^{2}|Y|^{4}\right\}
\end{aligned}
$$

The solution then becomes

$$
\psi(x, \tau)=\frac{1}{2 \epsilon} \exp \left[-i\left(\frac{k}{2 \epsilon} x-\frac{\omega \tau}{8 \epsilon^{2}} \tau\right)\right]\left[c-\frac{2 i c|\zeta|^{2} K W^{*} Y\left(G_{0}+c^{2} G_{2}\right)}{f_{30}+f_{32} c^{2}+f_{34} c^{4}}\right] .
$$

\section{LIMIT OF SASA-SATSUMA SOLITON SOLUTION}

To obtain this limit analytically we set $c=e^{-\rho}$ and shift accordingly the real part of the exponent $A$ by $\rho$, which moves the solution back to the origin thus removing the dependence of $A$ on $\rho$. Taking the limit $\rho \rightarrow \infty$ in Eq. (35) we obtain

$$
\psi(x, \tau)=-\frac{i}{\epsilon} \exp \left[A-\frac{i}{2 \epsilon}\left(k x-\frac{\omega \tau}{4 \epsilon}\right)\right] \frac{\Gamma \zeta^{*} Y^{*} \operatorname{Im}[\zeta]\left(e^{2 \operatorname{Re}[A]} \operatorname{Re}[\zeta]|\Gamma|^{2}+\zeta|Y|^{2}\right)}{\left(e^{4 \operatorname{Re}[A]} \operatorname{Re}[\zeta]^{2}|\Gamma|^{4}+2 e^{2 \operatorname{Re}[A]}|\Gamma \zeta Y|^{2}+\left|\zeta Y^{2}\right|^{2}\right)} .
$$

After a straightforward algebra this solution can be transformed into

$$
\psi(x, \tau)=\frac{-i a_{0} a_{1}}{2 \epsilon\left|\sqrt{b_{2} b_{3}}\right|} \exp \left[i \operatorname{Im}[A]-i\left(\frac{k}{2 \epsilon} x-\frac{\omega}{8 \epsilon^{2}} \tau\right)\right] \frac{2 \cosh (\operatorname{Re}[A])+\left(a_{2} / a_{1}-1\right) e^{-\operatorname{Re}[A]}}{\cosh \left(2 \operatorname{Re}[A]-\ln \left|\sqrt{b_{3} / b_{2}}\right|\right)+b_{1} /\left(2\left|\sqrt{b_{2} b_{3}}\right|\right)}
$$


with

$$
\begin{aligned}
a_{0} & =\Gamma \zeta^{*} Y^{*} \operatorname{Im}(\zeta), \quad a_{1}=\operatorname{Re}(\zeta)|\Gamma|^{2}, \quad a_{2}=\zeta|Y|^{2}, \\
b_{1} & =2|\Gamma|^{2}|\zeta|^{2}|Y|^{2}, \quad b_{2}=\operatorname{Re}\left([\zeta]^{2}\right)|\Gamma|^{4}, \quad b_{3}=|\zeta|^{2}|Y|^{4}, \\
\operatorname{Re}[A] & =\frac{\eta}{2 \epsilon}\left[x-\frac{\tau}{4 \epsilon}\left(\eta^{2}-3 \xi^{2}+\frac{1}{3}\right)\right], \\
\operatorname{Im}[A] & =\frac{\tau}{8 \epsilon^{2}}\left[\xi\left(3 \eta^{2}-\xi^{2}+\frac{1}{3}\right)+\frac{K}{3}\left(\frac{K^{2}}{9}-\frac{1}{3}\right)\right] \\
& +\frac{x}{2 \epsilon}\left(\frac{K}{3}-\xi\right) .
\end{aligned}
$$

We end up with the same solution (37) if we repeat the limiting process $c \rightarrow 0$ including all higher order terms $G_{4} c^{4}, G_{6} c^{6}$, $f_{36} c^{6}$, and $f_{38} c^{8} \neq 0$ in the expressions above. It is therefore the exact zero background $(c=0)$ limit of the solution (7) along the branch $\sigma=1, \sigma_{2}=1$. Thus, it is a particular exact solution of the SSE (1). It is illustrated in Fig. 7.

If we replace the real and imaginary parts of the eigenvalue using the following transformations:

$$
\eta=2 \epsilon \eta_{s} \quad \text { and } \quad \xi=\frac{1}{3}-2 \epsilon \xi_{s},
$$

and choose in particular for $\Gamma$ :

$\Gamma=\left[K-1+6 \epsilon\left(\xi_{s}-i \eta_{s}\right)\right] \frac{\left|1-6 \epsilon \xi_{s}\right|\left|1+6 \epsilon\left(i \eta_{s}-\xi_{s}\right)\right|}{3\left(1-6 \epsilon \xi_{s}\right)\left[6 \epsilon\left(\eta_{s}-i \xi_{s}\right)+i\right]}$,

we obtain the solution in a simpler form:

$$
\psi(x, \tau)=\eta_{s} e^{i B_{s}} \frac{2 \cosh A_{s}+\left(c_{s}-1\right) e^{-A_{s}}}{\cosh \left(2 A_{s}-\ln \left|c_{s}\right|\right)+\left|c_{s}\right|},
$$

where

$$
\begin{aligned}
A_{s} & =\eta_{s}\left\{x-\left[\xi_{s}+\epsilon\left(\eta_{s}^{2}-3 \xi_{s}^{2}\right)\right] \tau\right\}, \\
B_{s} & =\xi_{s}\left[x+\left(\frac{\eta_{s}^{2}-\xi_{s}^{2}}{2 \xi_{s}}+\epsilon\left(\xi_{s}^{2}-3 \eta_{s}^{2}\right)\right) \tau\right], \\
c_{s} & =1-\frac{i \eta_{s}}{\xi_{s}-\frac{1}{6 \epsilon}} ; \quad\left|c_{s}\right|=\sqrt{1+\frac{36 \eta_{s}^{2} \epsilon^{2}}{\left(1-6 \xi_{s} \epsilon\right)^{2}}} .
\end{aligned}
$$

Equation (40) represents the original version of the SasaSatsuma single-soliton solution to Eq. (1) with a small change

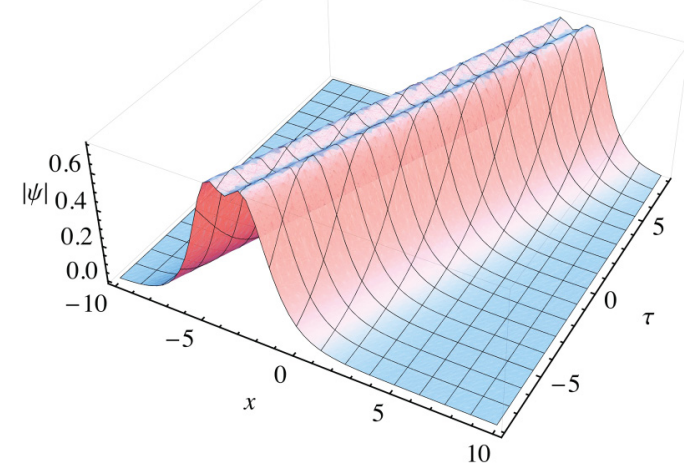

FIG. 7. (Color online) Amplitude profile of the solution (37) in the $(x, \tau)$ plane. Parameters chosen for this illustration are $\zeta=1 / 3+i$ ( $\xi=1 / 3, \eta=1), K=1.3(k=0.1), \epsilon=0.5$. According to Eq. (39) $\Gamma=-0.22136-i 0.98$. of notations. Namely, we added the subscript $s$ to the original parameters of Sasa-Satsuma in order to avoid confusion with other parameters of our work. The plus sign in front of $\epsilon$ in the expression for $A_{s}$ instead of the minus sign in the original Eq. (42) of the paper [2] is a correction of the typo. It is highlighted here by red color in the online version.

The solution (40) has the NLSE limit which is the fundamental soliton of the NLSE:

$$
\psi(x, \tau) \rightarrow \frac{\eta_{s} \exp \left[\frac{i}{2}\left(\eta_{s}^{2}-\xi_{s}^{2}\right) \tau+i \xi_{s}\left(x-x_{1}\right)\right]}{\cosh \left[\eta_{s}\left(x-x_{0}-\xi_{s} \tau\right)\right]}
$$

when $\epsilon \rightarrow 0$. In addition, the Sasa Satsuma solution has a singular limit

$$
\psi(x, \tau) \rightarrow \frac{4 \eta_{s}^{2} \exp \left[\left(2 \eta_{s}^{3} \epsilon+\frac{\eta_{s}}{6 \epsilon}\right) \tau+2 \eta_{s}\left(x+x_{0}\right)\right]}{\left[\exp \left(2 \eta_{s}^{3} \epsilon \tau+\frac{\eta_{s}}{6 \epsilon} \tau+2 \eta_{s} x_{0}\right)+2 \exp \left(2 \eta_{s} x\right)\right]^{2}}
$$

when $\left|c_{s}\right| \rightarrow \infty$. This happens when $\xi_{s} \rightarrow \frac{1}{6 \epsilon}$. This solution is singular either when $\epsilon \rightarrow 0$, or when $\epsilon \rightarrow \infty$. The velocity of the soliton (40) is given by

$$
v=\xi+\epsilon\left(\eta^{2}-3 \xi^{2}\right) .
$$

The plus sign in front of $\epsilon$ (red online) is a correction of the Sasa-Satsuma result. The velocity is infinite $v \rightarrow \infty$ when $\epsilon \rightarrow \infty$. On the other hand, the velocity goes to zero $v \rightarrow 0$ when

$$
\xi_{s} \rightarrow \frac{1 \pm \sqrt{12 \eta_{s}^{2} \epsilon^{2}+1}}{6 \epsilon}
$$

\section{CONCLUSIONS}

In this paper, we considered the simplest case of soliton on a background solution to the Sasa-Satsuma equation. This is just one branch of solutions of a complex manifold that has a relatively simple limit when the background amplitude goes to zero. Nevertheless, it has rich structure and admits several limiting cases that are important for applications. The zero background limit is complicated and admits known soliton solutions as well as singular solutions. Interestingly, solutions found by Mihalache and co-workers [8-10] have oscillating structure even with zero background. As such, they do not belong to the branch that we considered here. These other branches require a separate analysis and will be examined elsewhere.

\section{ACKNOWLEDGMENTS}

The authors acknowledge the support of the Australian Research Council (Discovery Project No. DP110102068). U.B. acknowledges the support from the Deutsche Forschungsgemeinschaft (DFG) within the collaborative research center MATHEON under Grant No. D14. N.A. also acknowledges the support from the Volkswagen Stiftung. N.A. acknowledges support through the Alexander von Humboldt Award (Germany). 
[1] V. S. Afrajmovich, V. I. Arnold, Y. S. Il'yashenko, and L. P. Shil'nikov, Dynamical Systems V (Springer-Verlag, Berlin, 2007).

[2] N. Sasa and J. Satsuma, J. Phys. Soc. Jpn. 60, 409 (1991).

[3] K. Trulsen and K. B. Dysthe, Wave Motion 24, 281 (1996).

[4] A. V. Slunyaev, J. Exp. Theor. Phys. 101, 926 (2005).

[5] M. J. Potasek, Phys. Lett. A 60, 449 (1991).

[6] S. B. Cavalcanti, J. C. Cressoni, H. R. da Cruz, and A. S. Gouveia-Neto, Phys. Rev. A 43, 6162 (1991).

[7] M. Trippenbach and Y. B. Band, Phys. Rev. A 57, 4791 (1998).

[8] D. Mihalache, L. Torner, F. Moldoveanu, N.-C. Panoiu, and N. Truta, J. Phys. A 26, L757 (1993).

[9] D. Mihalache, N.-C. Panoiu, F. Moldoveanu, and D.-M.Baboiu, J. Phys. A 27, 6177 (1994).

[10] D. Mihalache, L. Torner, F. Moldoveanu, N.-C. Panoiu, and N. Truta, Phys. Rev. E 48, 4699 (1993).

[11] M. Gedalin, T. C. Scott, and Y. B. Band, Phys. Rev. Lett. 78, 448 (1997).

[12] C. Gilson, J. Hietarinta, J. Nimmo, and Y. Ohta, Phys. Rev. E 68, 016614 (2003).

[13] J. Kim, Q. Han Park, and H. J. Shin, Phys. Rev. E 58, 6746 (1998).
[14] O. C. Wright III, Chaos Solitons Fractals 33, 374 (2007).

[15] M. Tajiri and Y. Watanabe, Phys. Rev. E 57, 3510 (1998).

[16] N. Akhmediev, J. M. Soto-Crespo, and A. Ankiewicz, Phys. Lett. A 373, 2137 (2009).

[17] U. Bandelow and N. Akhmediev, Phys. Lett. A 376, 1558 (2012).

[18] N. Akhmediev and A. Ankiewicz, Solitons: Nonlinear Pulses and Beams, Optical and Quantum Electronics No. 5, Chaps. 3 and 4 (Chapman \& Hall, London, 1997).

[19] N. Akhmediev and S. Wabnitz, J. Opt. Soc. Am. B 9, 236 (1992).

[20] M. Erkintalo, G. Genty, B. Wetzel, and J. M. Dudley, Phys. Lett. A 375, 2029 (2011).

[21] C. Mahnke and F. Mitschke, Phys. Rev. A 85, 033808 (2012).

[22] N. Akhmediev and V. I. Korneev, Theor. Math. Phys. 69, 1089 (1986) [Teor. Mat. Fiz. (USSR) 69, 1089 (1986)] .

[23] B. Kibler, J. Fatome, C. Finot, G. Millot, G. Genty, B. Wetzel, N. Akhmediev, F. Dias, and J. M. Dudley, Sci. Rep. 2, 463 (2012).

[24] N. Akhmediev, A. Ankiewicz, and M. Taki, Phys. Lett. A 373, 675 (2009).

[25] A. Ankiewicz, J. M. Soto-Crespo, and N. Akhmediev, Phys. Rev. E 81, 046602 (2010). 